\title{
Tratamientos basados en incretinas y mortalidad en pacientes con diabetes tipo 2
}

\author{
Incretin based treatments and mortality in patients with type 2 diabetes
}

Jiali Liu y col. BMJ 2017;357:j2499.

\section{Objetivos}

Comparar el efecto del tratamiento con incretinas (agonistas GLP-1 o inhibidores DPP-4) contra placebo, modificaciones del estilo de vida o contra drogas anti-hiperglucemiantes en la mortalidad de los pacientes con diabetes tipo 2.

\section{Fuente de datos}

Se realizó una búsqueda en Medline, Embase, en el Registro de Ensayos Clínicos Controlados de Cochrane (CENTRAL) y en ClinicalTrials.gov para identificar estudios relevantes hasta febrero de 2017, sin restricciones de lenguaje. Fueron usados términos $\mathrm{MeSH}$ y en texto libre.

\section{Selección de estudios}

Fueron incluidos ensayos clínicos controlados en pacientes con diabetes tipo 2 con un seguimiento mayor o igual a 12 semanas y un reporte explícito de mortalidad por todas las causas, que hubieran comparado agonistas GLP-1 o inhibidores de DPP-4 contra placebo, modificaciones del estilo de vida o contra drogas anti-hiperglucemiantes.

\section{Extracción de datos}

Dos revisores independientes extrajeron los datos y evaluaron el riesgo de sesgo. Las discrepancias fueron resultas mediantes discusión conjunta o a través de la participación de un tercer revisor.

\section{Resultados principales}

Fueron incluidos 189 ensayos clínicos controlados aleatorizados $(n=155.145)$, con un riesgo bajo a moderado de parcialidad; 77 no informaron eventos de muerte y 112 informaron 3.888 muertes en 151.614 pacientes. Representaron el $97 \%$ del peso del análisis los estudios que habían comparado agonistas GLP-1 e inhibidores de DPP-4 con placebo o ningún tratamiento. Ver tabla 1. No se observaron diferencias en la mortalidad por todas las causas entre los pacientes asignados a tratamiento con incretinas vs. control (1925/84136 vs 1963/67 478; OR 0,96; IC95\% 0,90 a

Tabla 1: Mortalidad por todas las causas en pacientes con diabetes tipo 2 con tratamiento con incretinas versus placebo

\begin{tabular}{l|c|c|c|c|}
\hline \multirow{2}{*}{ DROGAS } & \multicolumn{4}{|c|}{ Número de muertes/total } \\
\cline { 2 - 5 } & Incretina & Placebo & OR (IC95\%) & 0,03 \\
\hline A GLP-1 & $654 / 9350$ & $730 / 9355$ & $0,89(0,80$ a 0,99$)$ & 0,74 \\
\hline I DPP-4 & $1120 / 18313$ & $1088 / 18230$ & $1,02(0,91$ a 1,14) & 0,48 \\
\hline Total (A GLP-1 + I DPP-4) & $1174 / 27663$ & $1818 / 27585$ & $0,97(0,88$ a 1,06) & 0.40 \\
\hline
\end{tabular}

A GLP-: análogos GLP-1. I DPP-4: inhibidores DPP-4. OR: Odds Ratio. p: p valor efecto total test $z$

1,02). El grupo tratado con incretinas tuvo tres eventos cardiovasculares menos (IC 95\% 1 a 7 ) cada mil pacientes tratados durante cinco (evidencia de calidad moderada). Si bien los resultados de los análisis de subgrupos son inestables, se observó una tendencia ligeramente favorable hacia los agonistas de GLP-1 pero no a los inhibidores de DPP-4.

\section{Conclusiones}

No hay evidencia por el momento de que el tratamiento con incretinas incremente la mortalidad por todas las causas en pacientes con diabetes tipo 2. Se necesitan estudios aleatorizados bien diseñados para confirmar o refutar definitivamente las posibles diferencias en el efecto de los agonistas GLP-1 y de los inhibidores de la DPP-4 en la mortalidad por todas las causas.

Fuente de financiamiento/conflicto de interés: National Natural Science Foundation of China. Los autores no comunicaron conflicto de interés.

\section{COMENTARIO}

Los análogos de GLP-1 y los inhibidores de DPP-4 representan importantes opciones para el tratamiento de los pacientes con diabetes tipo $2^{1}$. Se utilizan como monoterapia en aquellas situaciones en las que la metformina no es tolerada, y además, en combinación con otros agentes antihiperglucemiantes en el diagnóstico de la enfermedad o ante su progresión. La controversia en la bibliografía sobre la asociación entre el uso de incretinas y la mortalidad ${ }^{2-6}$, motivó la realización de este metanálisis, que concluyó que por el momento no hay evidencia de que el trata-miento con incretinas incremente la mortalidad por todas las causas en pacientes con diabetes tipo 2 . Sin embargo, algunos estudios incluidos en éste meta-análisis podrían informar selectivamente los datos con respecto a la muerte en sus publicaciones completas, con el consiguiente riesgo de sesgo de selección. Otra debilidad de este metanálisis fue la incapacidad de evaluar los efectos a largo plazo, ya que entre los 189 ensayos, solo 40 tuvieron un seguimiento mayor a 52 semanas (el seguimiento más prolongado fue de 3,8 años).

\section{Conclusiones de la comentadora}

Las incretinas están vigentes en los estándares de tratamiento de varias Sociedades Científicas ${ }^{1,7-9}$. Es importante adecuarse a los mismos y tener en cuenta las particularidades de cada paciente al momento de decidir la terapia farmacológica. Los pacientes con diabetes tipo 2 deben ser periódicamente evaluados a fin de prevenir y controlar las complicaciones que puedan aparecer tanto en la evolución natural de la enfermedad como secundarias al tratamiento farmacológico.

Vanina Pagotto [Departamento de Investigación, Hospital Italiano de Buenos Aires. vanina.pagotto@hospitalitaliano.org.ar]

Pagotto, V. Tratamientos basados en incretinas y mortalidad en pacientes con diabetes tipo 2. Evid Act Pract Ambul. 2018; 21(1):35. Comentado de: Jiali Liu y col. Incretin based treatments and mortality in patients with type 2 diabetes: systematic review and meta-analysis. BMJ. 2017 Jun 8;357:j2499. PMID: 28596247.

\section{Referencias Bibliográficas}

1.Nauck M. Incretin therapies: highlighting common features and differences in the modes of action of glucagon-like peptide-1 receptor agonists and dipeptidyl peptidase-4 inhibitors. Diabetes Obes Metab. 2016 Mar;18(3):203-16.

2.Scirica B y col. Bhatt DL, Braunwald E, Gabriel Steg P, Davidson J, Hirshberg B, et al. Saxagliptin and Cardiovascular Outcomes in Patients with Type 2 Diabetes Mellitus. N Engl J Med. 2013;369(14):1317-26.

3.Green JB, y col. Effect of Sitagliptin on Cardiovascular Outcomes in Type 2 Diabetes. N Engl J Med. 2015;373(3):232-42.

3.Green JB, y col. Effect of Sitagliptin on Cardiovascular Outcomes in Type 2 Diabetes. N Engl J Med. 2015;373(3):232-42.
4.White WB, y col. Alogliptin after acute coronary syndrome in patients with type 2 diabetes. N Engl J Med. 2013 Oct 3;369(14):1327-35.

5.Mogensen UM, y col Cardiovascular safety of combination therapies with incretin-based drugs and metformin compared with a combination of metformin and sulphonylurea in type 2 diabetes mellitus--a retrospective nationwide study. Diabetes Obes Metab. 2014 Oct;16(10):1001-8.

6.Giorda CB, y col. Hospitalisation for heart failure and mortality associated with dipeptidyl peptidase 4 (DPP-4) inhibitor use in an unselected population of subjects with type 2 diabetes: a nested case-control study. BMJ Open. 2015;5(6):e007959-e007959.

7.American Diabetes Association. Standards of Medical Care in Diabetes -2017. Diabetes Car. 2017 Jan;40(1).

8.Guías para el tratamiento de la diabetes mellitus tipo 2. Sociedad Argentina de Diabetes. Revista de la Sociedad Argentina de Diabetes. 2016 Aug;50(2).

9.Garber A, y col. Consensus Statement By The American Association Of Clinical Endocrinologists And American College Of Endocrinology On The Comprehensive Type 2 Diabetes Management Algorithm - 2018 Executive Summary. Endocrine Practice. 2018 Jan;24(1). 